\title{
VLBI TIME-TRANSFER USING CONT08 DATA
}

\author{
Carsten Rieck $^{(1,2)}$, Rüdiger Haas ${ }^{(2)}$, Kenneth Jaldehag ${ }^{(1)}$, Jan Johansson ${ }^{(1,2)}$ \\ (1) SP Technical Research Institute of Sweden \\ Box 857, SE-50115 Borås, Sweden \\ +46 (0)105-165000 \\ carsten.rieck@sp.se \\ kenneth.jaldehag@sp.se \\ jan.johansson@sp.se \\ (2) Chalmers University of Technology, Onsala Space Observatory \\ SE-439 92 Onsala, Sweden \\ +46 (0)31-7725500 \\ rudiger.haas@chalmers.se
}

\begin{abstract}
One important prerequisite for geodetic Very Long Baseline Interferometry (VLBI) is the use of frequency standards with excellent short term stability, i.e. hydrogen masers. This makes VLBI stations, which are often co-located with Global Navigation Satellite System (GNSS) receiving stations, interesting for studies of time- and frequency-transfer techniques. In this paper we present an assessment of VLBI time-transfer based on the data of the two week long consecutive IVS Cont08 VLBI-campaign by using GPS Carrier Phase (GPSCP). Cont08 was a 15 days long campaign in August 2008 that involved eleven VLBI stations on five continents. For Cont08 we estimated the worst case VLBI time link stability between the station clocks of ONSALA and WETTZELL to about 1.5e-15 at one day. Comparisons with clock differences estimated with GPSCP confirm the VLBI results. The paper also indicates time-transfer related problems of the VLBI technique as used today.
\end{abstract}

\section{INTRODUCTION}

Despite the fact that Very Long Baseline Interferometry (VLBI) has been around for about 40 years, recent information on the use of VLBI for time/frequency transfer is sparse. Many early investigations were driven by the need of the Deep Space Network (DNA) [1] ground station time synchronization. Link instabilities of 1e-14@1d were reported and ns accuracy of time-transfer was verified by clock transport experiments with uncertainties of about 5 ns [4]. The importance of calibration was identified and attempts were made to calibrate instrumental delays [5]. References [7-9] use sophisticated differential station calibration techniques. During recent years the National Institute of Information and Communications Technology (NICT) has raised interest in using VLBI for time-transfer applications. Work done in [11] resulted in frequency link instabilities surpassing GPS Carrier Phase (GPSCP) with better than 1e-15@1d modified Allan deviation (MDEV). Table 1 summarizes some historical key results.

Even though the calculus for analysis of both VLBI and Global Navigation Satellite System (GNSS) data is very similar, time comparisons using VLBI can be seen as an independent technique and thus, in the future, can be an alternative option for international time-transfer. The development of the next generation VLBI system, called VLBI2010, might allow making VLBI time-transfer operational. Traditional long distance time-transfer methods, like GNSS and Two Way Satellite Time and Frequency Transfer (TWSTFT), are often heavily dependent on active third parties that are not necessarily dependable in the long term. The two named satellite-based techniques are today the backbone of international time metrology and are used for the formation of the international time scale UTC, Universal Time Coordinated. These techniques can fast become outdated due to economical or political reasons. A passive method based on VLBI technology could become a major time-transfer technique if the development of small equipment is forced. The intent of this paper is to reproduce and confirm results reported before and gain a better understanding of VLBI in terms of the system delays. An additional motivation is the fact that some of the IVS sites maintain UTC realizations and therefore the establishment of an alternative and independent time-transfer method based on VLBI would be useful.

\section{SHORT SUMMARY OF THE VLBI TECHNIQUE}

VLBI is an interferometry concept designed as a radio astronomy tool in the 1950ies. But not before the late sixties the first observational components were developed in the US/Canada resulting in the first transatlantic VLBI session that was carried out as a pioneer experiment between the telescopes at Westford in the USA and the Onsala Space 
Table 1. A non-comprehensive chronological list of previous publications in the field of time- and frequency transfer using VLBI. The early references are associated with the synchronization within the Deep Space Network using VLBI. The last column specifies some key results.

\begin{tabular}{|l|l||l|l|}
\hline Author & REF & Year & Key Results \\
\hline Clark et al. & {$[2]$} & 1977 & ns accuracy, equipment delay calibration \\
\hline Hurd et al. & {$[3]$} & 1978 & 1 e-13 link stability, 1e-14 possible \\
\hline Spencer et al. & {$[4]$} & 1981 & 5 ns in time, rate uncertainty 1.3e-14 \\
\hline \hline Johnston et al. & {$[5]$} & 1983 & MARK III calibration, sub-nanoseconds \\
\hline Ward & {$[6]$} & 1984 & Clock synchronization using VLBI \\
\hline Hama et al. & {$[7]$} & 1989 & Zero baseline calibration $($ ZBI) with ns accuracy \\
\hline Yoshino et al. & {$[8,9]$} & 1994 & Short baseline (SBI) calibration, precision $<1 \mathrm{~ns}$ \\
\hline Takiguchi et al. & {$[10,11]$} & 2007,2008 & Frequency link uncertainty $<1 \mathrm{e}-15 @ 1 \mathrm{~d}$ \\
\hline
\end{tabular}

Observatory (OSO) in Sweden in 1968. Advances in technology are numbered starting with the MARK I system in the 70ties to the MARK V of today. The development of equipment in North America was also followed in Japan that developed its own compatible ' $\mathrm{K}$ ' series systems. A leap in technology was achieved with the MARK III in the 1980ies with major parts of this equipment still in use today. Current development towards VLBI2010 will enhance both hardware and analysis strategies towards higher bandwidths, higher sensitivity and faster result turnaround times.

Single dish radio astronomy is limited by the poor angular resolution of microwave telescopes compared to that of optical instruments. A baseline interferometer is based on the estimation of the differences of the arrival times of planar wave fronts at two locations in order to gain resolution in the observations. Because angular resolution $\theta$ is inversely proportional to antenna aperture, a separation $D$ of two antennas that simultaneously observe the same object creates a large synthetic aperture with a resolution of about $\theta=\lambda / D$ where $\lambda$ is the wavelength of the observation. Thus a large separation of the antennas yields a superior resolution that can be used to image structures of astronomical objects. The method was fast adapted by geodesy that uses the VLBI concept in an orthogonal sense by observing inertial point radio sources, which makes estimation of station positions possible. Geodetic VLBI is mainly used for maintenance of reference frames binding celestial and terrestrial frames together. Further it is a scientific tool for the study of geodynamic processes, earth rotation and orientation, plate tectonics and rebound effects and it serves as well for estimation of troposphere and ionosphere parameters.

Fig. 1 shows a simplified equivalent signal model of VLBI. An arbitrary time depended source signal is split into two paths with one path experiencing an additional delay $\tau$. Because the noise terms added to each signal path are considered uncorrelated the source signal can be separated during the correlation process. The noise is the combined site dependent disturbances of the source signal. Geometrically the scenario is depicted in Fig. 2.

Radio sources used in geodetic VLBI are extragalactic quasars, in the order of million of light years (Mpc), and other similar compact celestial bodies. A VLBI experiment is divided into scans where a radio source is observed during a period of time. The scan length is dependent on the telescopes sensitivity and on the flux density of the source. During a typical $24 \mathrm{~h}$ session up to 300 sources are used, which are chosen as homogeneously distributed over the hemisphere as possible in order to provide a good geometry.

Geodetic VLBI of year 2010 is able to receive within $500 \mathrm{MHz}$ in the S- and X-bands, where the combination of group delay observations is used to compensate for the dispersing effects of the ionosphere. The bands are divided into six channels in S- and four each in the lower and upper X-band. Each channel has a configurable baseband bandwidth of 2 to $16 \mathrm{MHz}$ and the upper or/and lower sidebands of each channel are sampled according to Nyquist with one bit resolution. Usually 16 channels can be recorded on to digital media with a maximal data rate of $512 \mathrm{Mbit} / \mathrm{s}$. This results into up to 5 Tbyte of data for a 24 hour session.

Because the characteristic of the signals emitted by radio sources is not known, the systems need predictable local clocks that can meet certain stability requirements. This is partly due to the need of stable conditions during a single scan and is also needed for connecting the different arcs during one session. Typical clock stabilities required are about 1e-13@100s and 3e-15@10000s, which mostly implies the use of active hydrogen masers.

The geometrical delay between two receivers is defined by the vector product between the baseline $D$ and the vector $\hat{i}_{s}$ to the radio source. VLBI derived delays are affected by the geophysics of the earth, the atmosphere and relativity. But it is the phase and frequency offset of the station clocks that dominate the deviation from the theoretical delay between two stations. Today, equipment and modeling allows the estimation of delay values with uncertainties in the order of $10 \mathrm{ps}$, which is not yet good enough to reach $1 \mathrm{~mm}$ precision for reference frame applications. Currently, most potential 
for improvement can be seen at the equipment side of VLBI, which has been addressed by VLBI2010 with an improved bandwidth and more sensitive receiver systems [12-13].

$$
\begin{gathered}
\tau_{g}=-\frac{D \cdot \hat{l}_{s}}{c} \\
\sigma_{\tau} \approx \frac{1}{2 \pi \cdot S N R \cdot B^{*}} \approx 10 p s \\
S N R \approx \sqrt{\frac{A_{1} \cdot A_{2}}{T_{1} \cdot T_{2}} \cdot 2 \cdot B^{*} \cdot t} \cdot \frac{F}{2 k} \approx 30
\end{gathered}
$$

The signal to noise ratio is dependent on the sensitivity of the antenna systems of a baseline, i.e. the combined dish areas $A$ and their system temperatures $T$; the observed synthesized bandwidth $B^{*}$, integration time $t$ and the radio source flux density. Equations (1) to (3) define rough relations. Typical receiving systems exhibit system temperatures of about 50-100 K absolute temperature and antenna diameters of 20-60 m. This corresponds to system equivalent flux densities (SEFD) in the order of about 100-1500 Jy, whereas radio sources observed in geodetic VLBI have flux densities in the order of 1-3 Jy.

Traditionally, the disk recorded data are physically shipped to a correlator station that does the experiment correlation. VLBI data transport using communication networks, such as IP, is called e-VLBI and allow correlation and analysis in real-time. This usually requires data rates in the proximity of $1 \mathrm{Gbit} / \mathrm{s}$ for typical experiments. The correlation process is a complex data reduction scheme with a compression ratio in the order of $1 \mathrm{e} 9$. It produces so called fringes that are iteratively searched in order to find the group delay between two stations. Bandwidth synthesis combines the different channels in each band by applying the phase delay calibration system (PCAL) that is used to calibrate the signal path at each station. The correlation results in pairs of group delay and group delay rate values per baseline, scan and band (X and S). In a subsequent step the correlation output is input to a baseline- or network analysis. There are a number of analysis strategies available such as algorithms using least-squares (LSQ), least-squares collocation (LSQC), Kalman filtering (KF) or square-root information filters (SRIF). The Calc/Solve software package [14] that is used by several IVS analysis centres is based on least-squares (LSQ). It estimates the model deviations of all parameters of interest. The station clock differences are usually modelled using quadratic terms with constrained deviation estimates typically sampled every hour. As with the correlation the baseline analysis is usually done in 24 hour batches.

\section{TIME AND FREQUENCY WITHIN THE VLBI SYSTEM}

Fig. 3 depicts a typical setup of a time and frequency distribution in the VLBI back-end. The frequency source is usually a hydrogen maser that supplies the local oscillators (LO) of the front-end, the PCAL system and the video converters (VC) with a $5 \mathrm{MHz}$ reference frequency. The time stamping of the data recording is governed by the time scale that is maintained by the formatter clock, which is the only interface to a local time scale, like a UTC(k). Often the formatter clock has a 1PPS output that is used for monitoring the clock offset during the experiment using a GPS code receiver. In order to use VLBI as a time-transfer system with practical value at least three items on the equipment side have to be assured:

1. The formatter clock has to be locally calibrated and constantly measured against its source clock 1PPS, which should result in a constant offset. Another possibility would be to retain a reference delay measurement system to



Fig. 1. Equivalent signal model of VLBI.

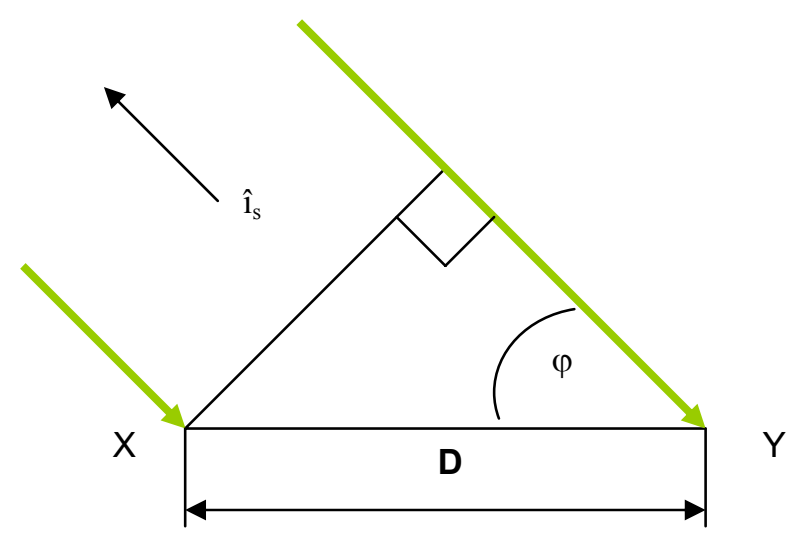

Fig. 2. VLBI geometry. 


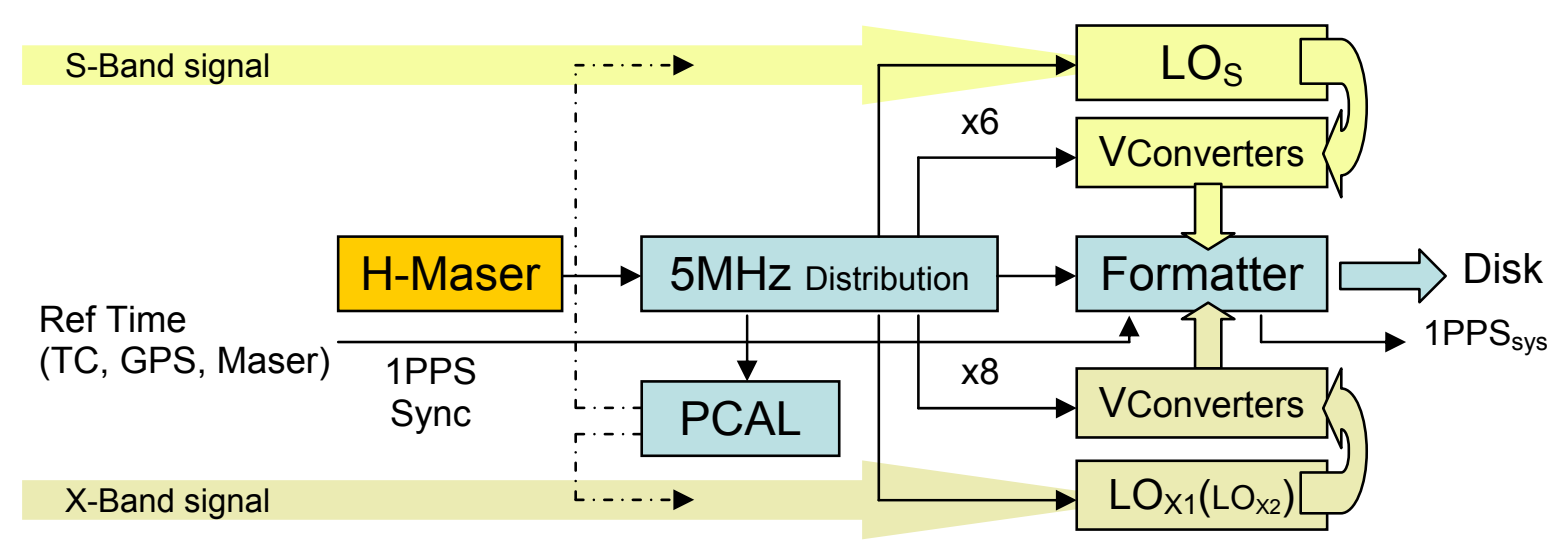

Fig. 3. Timing of the VLBI backend. An H-maser frequency distribution supplies all equipment with coherent frequency. The formatter clock defines the time representation for the VLBI processing by providing an arbitrary synchronized timescale to the disk recording. The PCAL generates a drain of short pulses $1 \mu$ s apart that is injected into the $\mathrm{X}$ and $\mathrm{S}$ band feeds close to the antenna. The signal is visible as tones spaced $1 \mathrm{MHz}$ apart and propagates through all the entire system. Video conversion is done on $1 \mathrm{MHz}$ boundaries such that the PCAL signal is present as a $10 \mathrm{kHz}$ tone in each recorded channel. Today the PCAL is used during the correlation process and is then lost, however, in a timing application it has the potential to be used to calibrate and/or maintain a calibration of the receiver chain, but this is unfortunately unexploited so far.

another clock or timescale, such as a UTC(k) representation.

2. The signal chain from the fiducial point of the antenna system to the formatter clock needs to be calibrated. This calibration is difficult and has been attempted by measuring equipment delays, clock transport experiments and differential calibrations using portable VLBI stations.

3. Common mode delays induced by slow environmental changes (i.e. temperature variations) propagate directly into the estimation of the local clock difference of a baseline. VLBI uses a phase calibration system (PCAL) for synthesising a larger bandwidth by measuring small portions of the entire band. This system could also be used to monitor and maintain the calibration of the receiver chain.

Geodetic driven VLBI is usually not interested in the clock parameter and thus all precautions according to the above list are usually neglected because they do not affect other parameters of interest. Fig. 4 presents a measurement of the Onsala formatter clock relative to its source clock during 88 days in 2010 using a 50 ps time interval measurement system. Apart from the obvious but undetermined erratic $1 \mathrm{~ns}$ instability the time series also shows unexplained repetitive patterns every 75000-81000 s. Compared to the local timescale the formatter output experiences several times as much noise as the source clock at any time interval $\tau$. Typical RMS values for periods without time jumps are around 100 ps. It is not clear if the formatter 1PPS represents the noise that is apparent in the recording time stamps, but if the time stamping is noisy at the $500 \mathrm{ps}$ level it will limit the time-transfer capabilities of VLBI. For the continuous part in Fig. 4 the Allan deviation is about 2.6e-15@1d, which is not limited by the time interval counter (TIC), but may contain considerable noise from the auxiliary output generator (AOG) based UTC timescale.

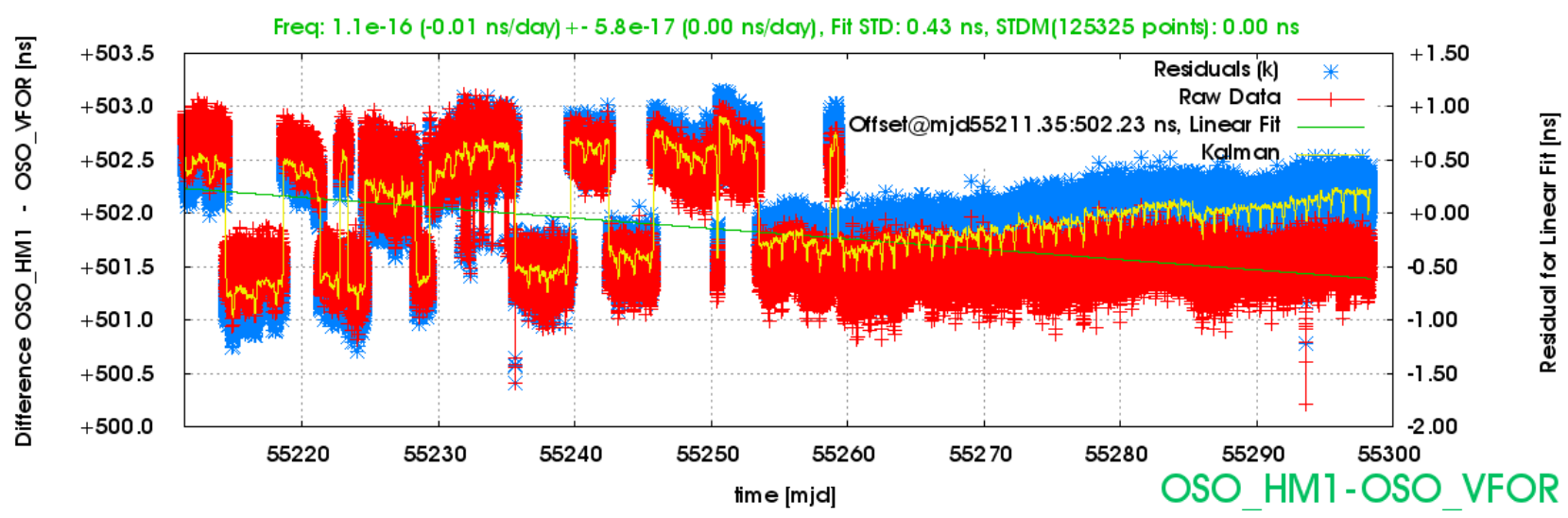

Fig. 4. Onsala VLBI system formatter 1PPS measured relative to its source clock 1PPS during an 88 day period in 2010 . Red shows the measured offset values, whereas blue shows the residuals to a linear fit of the data. Yellow depicts a Kalman filtered time series of the residuals. The experienced offset of about $500 \mathrm{~ns}$ is reasonable in value, but is not calibrated. The time difference is calculated by differencing sequential measurements to the local UTC timescale every $60 \mathrm{~s}$, with individual data points measured $12 \mathrm{~s}$ apart. 


\section{THE CONT08 SESSION OF THE IVS}

The continuous sessions of the IVS [15] have been a tool for the assessment of the VLBI technique and are of special scientific value. The Cont08 experiment was carried out during August 12 and August 26 in 2008 involving eleven stations on five continents using a $512 \mathrm{Mbit} / \mathrm{s}$ recording. Besides its main scientific goal, the study of high frequent sub-diurnal earth orientation parameters (EOP), it was also used to asses the improvement of the VLBI technique and its accuracy, and to make comparative studies with co-located techniques like GNSS, SLR and DORIS [16]. Fig. 5 shows the station distribution of Cont08. The data of the experiment were correlated in $24 \mathrm{~h}$ batches by the Washington correlator

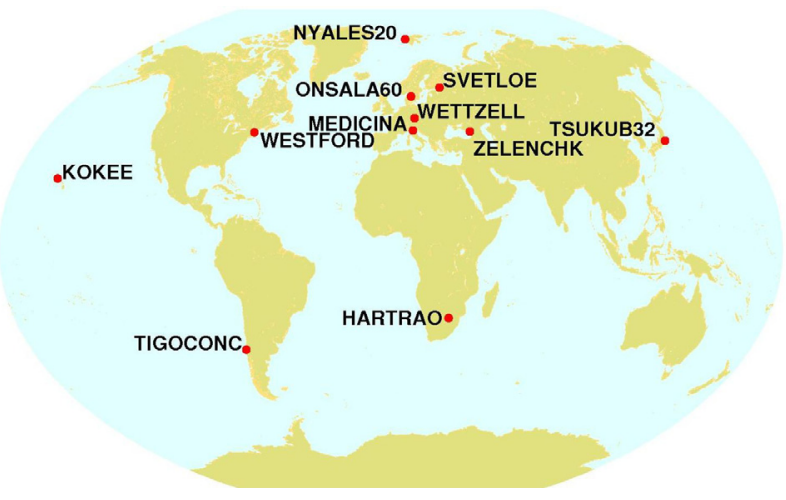

Fig. 5. Stations of the IVS Cont08 experiment. From [14]. (WACO) using KOKEE as its reference.

\section{VLBI TIME TRANSFER, NETWORK ANALYSIS (NA)}

For initial tests we applied the basic CALC/SOLVE network processing setup (NA) used at Onsala for processing the regular EOP sessions. The data was processed in batches of 24 hours each where the clock difference parameter was estimated as a quadratic term with deviation estimates every hour. The batch processing gives rise to discontinuities at the day boundaries, which affects all parameters that are continuous at day boundaries. Day boundary jumps are generally expected to be in the order of the uncertainties of the estimates. Fig. 6 shows the estimated discontinuities during Cont08. The last two days of the experiment, MJD 54703 and 54704, show excessive offsets of about 50 ns with an almost inverted behaviour between the two days. The magnitudes of these offsets are not explainable from the modelling and are believed to be an effect of the correlation process. From a geodetic point of view additional biases are no problem as long as they are constant during one common period of correlation and analysis. It is clear that these disturbing conditions, i.e. a) discontinuities through analysis, and b) biases during correlation, make it impossible to use the results in an absolute time-transfer. Random correlator biases might even prevent consistent calibration efforts.

The output of the Calc/Solve analysis allows concatenating two adjacent solutions because of overlapping estimates. This removes any meaning of a phase bias, but can be useful for evaluation of a frequency-transfer. Day boundary jumps introduced by analysis seem not to be biased, thus errors to the estimated frequency due to their removal should be small, but will exist. The standard processing uses the KOKEE station as its clock reference estimating all other station clocks relative to it. Throughout Cont08 KOKEE experienced an excessive diurnal signature in its local clock likely caused by environment, see Fig. 7. Thus, we changed the processing to use ONSALA60 as the reference clock in order to more easily asses the transfer results. This of course does not change the fact that the correlation is based on a badly behaving clock, which can be one reason for correlator introduced biases. Fig. 8 shows the best case of a relative VLBI time-transfer during Cont08, between the stations of Onsala and Wettzell.

The processing allows three degrees of freedom regarding the clock parameter: 1. linear or quadratic clock modelling, 2. constraining of the variance of the clock difference frequency, and $\mathbf{3}$. choice of sampling interval of the estimation of the clock deviation from its model. For the purpose of this paper we used quadratic modelling and we varied the constraints and the sampling in order to find a reasonable setup. In the standard setup the clock parameters are loosely constrained, 1e-13 at 1 hour sampling, in order to allow the absorption of effects caused by physical phenomena that are not properly modelled, such as certain loading types or errors in the atmospheric delay mapping. As the main interest lies in the estimation of clock differences the actual clock constraints should be adjusted to the stability of the involved station clocks. This in turn will result in a better clock estimation, but will likely increase the magnitude of the residuals. For example, the Allan deviation of a frequency drift removed phase measurement between a SigmaTau and a CH1-75A maser at SP is better than 5e-15 for time intervals of 20-60 minutes, but unfortunately Allan stabilities cannot be directly translated to SOLVE type constraints. A setup with $1200 \mathrm{~s}$ sampling and a moderate 5e-14 constraint was used to generate Allan stabilities presented in Fig. 9.

\section{VLBI SINGLE BASELINE ANALYSIS (SBA)}

Because of its good performance in the network case we used the ONSALA60-WETTZELL baseline in a single baseline analysis in order to study the effect of constraints for a $1200 \mathrm{~s}$ sampling interval. The phase difference between the network and single baseline solution for the same constraint is significant and can be seen as a frequency offset of about 50ps per day. Even the different SBA/NA solutions differ, most noticeable by phase jumps at the day boundaries 

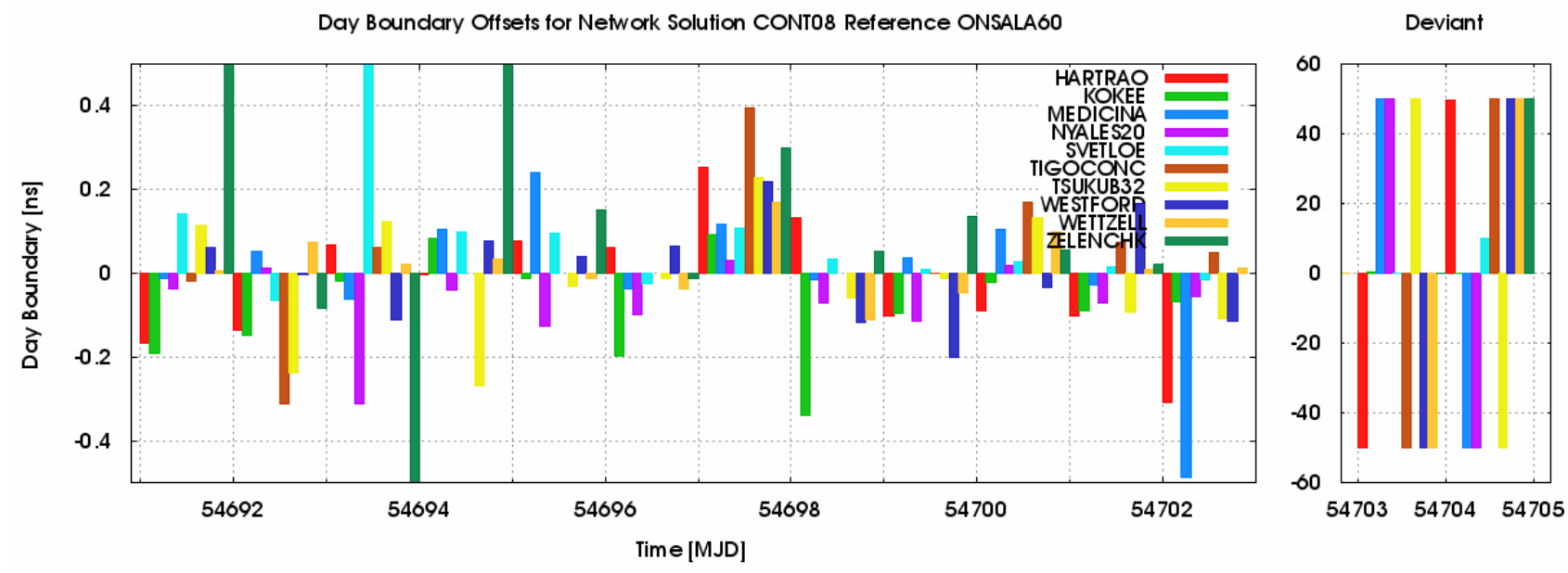

Fig. 6. Day boundary offsets during the Cont08 experiment using a Calc/Solve network analysis. The y axis of the left part is clipped, not showing excessive day boundary jumps for ZELENCHK and SVETLOE $>>1$ ns. Note the different scale for the right plot. Formal uncertainties of the clock estimates are in the order of $30 \mathrm{ps}$.



Fig. 7. KOKEE clock diurnal signature. Blue depicts the second order detrended and bias removed clock offset between KOKEE and ONSALA60 which exhibits an excessive diurnal signature. The time series is anti-correlated with the outside temperature at the KOKEE station (in red) and correlated with the temperature at Onsala (in green). The same variations can be seen in the GPS carrier phase PPP solutions for the IGS KOKB and thus can be attributed to KOKEE. KOKEE is used during correlation of the Cont08 experiment, which might have caused biases due to the bad clock.

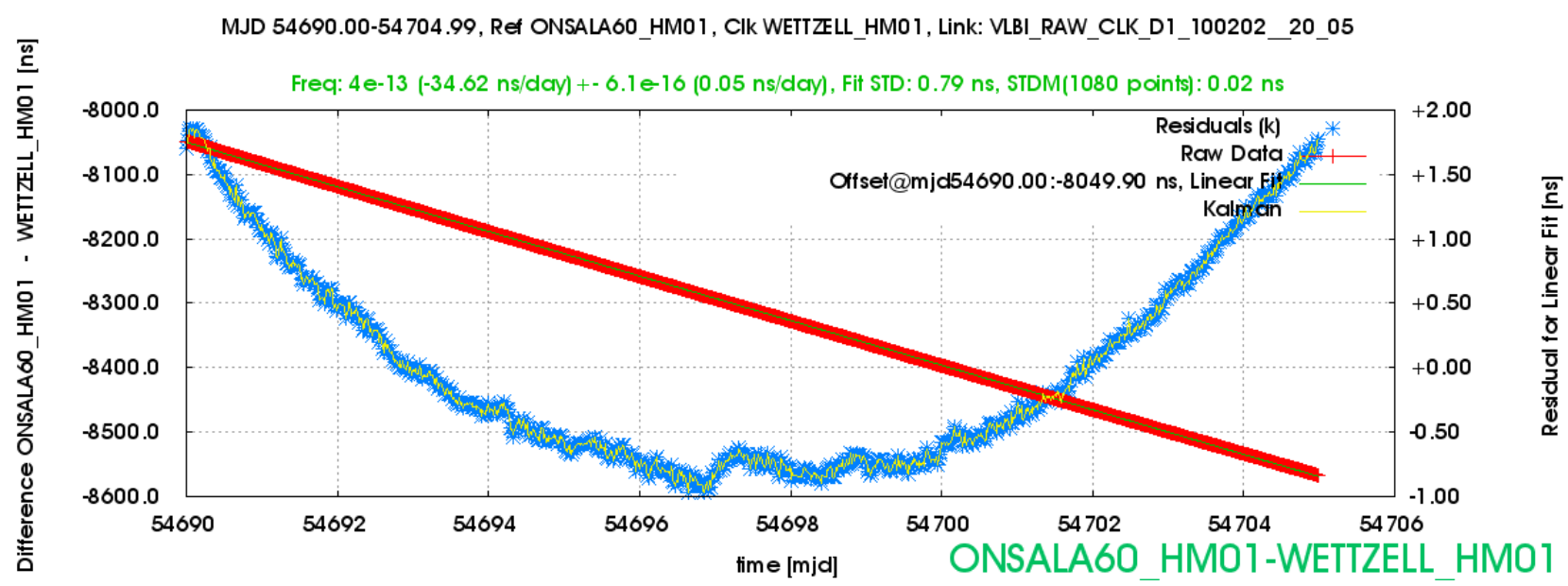

Fig. 8. VLBI Time-transfer ONSALA60-WETTELL during Cont08 using CALC/SOLVE after removal of day boundary biases. The example is based on a clock parameter estimation sampled every 20 minutes using a frequency constraint of $5 \mathrm{e}-14$ where the uncertainty in the estimated time offset is unknown. The Allan deviation of the $2^{\text {nd }}$ order detrended time series shows a frequency stability of about $1.2 \mathrm{e}-15$ at time intervals of one day. 


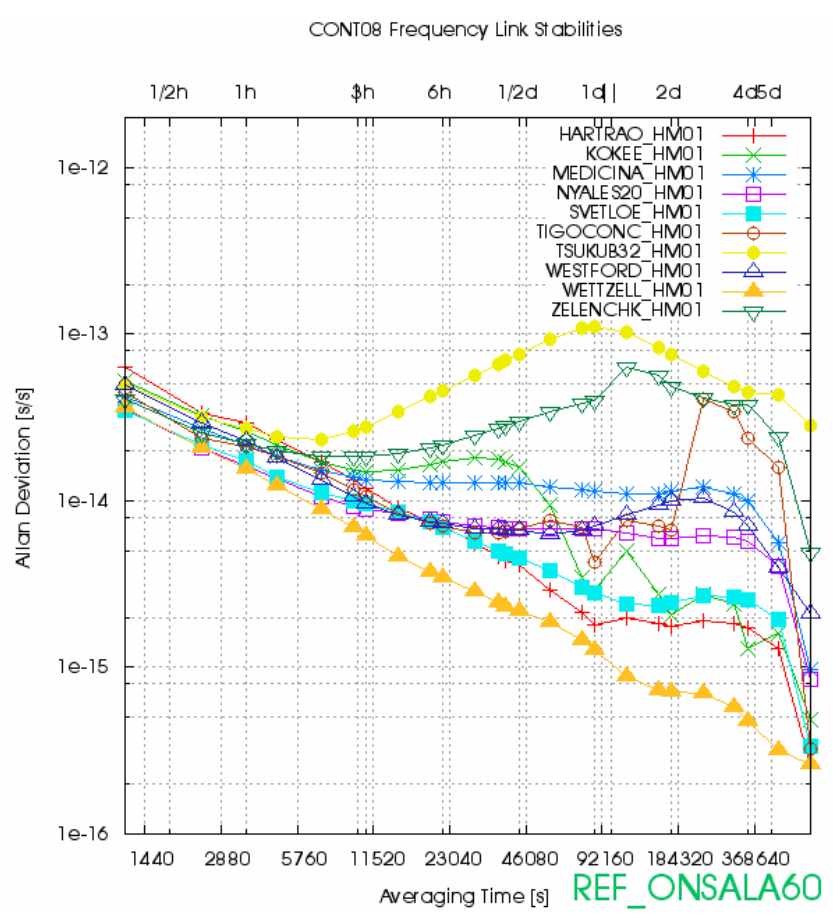

Fig. 9. Cont08 VLBI derived frequency stabilities (ADEV) of $2^{\text {nd }}$ order detrended CALC/SOLVE network solutions for the $1200 \mathrm{~s}$ sampling and 5e-14 constraint case. The TSUKUBA station clock experienced a dramatic frequency change during the last few days of the experiment.



Fig. 10. Baseline analysis of the ONSALA60 WETTZELL baseline using a $1200 \mathrm{~s}$ sampling and different clock constraints. The network solutions behave slightly worse than their single baseline counterparts.

with their magnitude roughly proportional to the constraint difference. Fig. 10 shows the Allan deviations of different $2^{\text {nd }}$ order detrended solutions. Constraints harder than $5 \mathrm{e}-14$ seem to create problems in the processing where the clock performance is overestimated and the LSQ analysis cannot separate the clocks from instrumental noise.

\section{GPS CARRIER PHASE COMPARISONS}

All IVS stations have co-located IGS receivers, where seven out of eleven used a common clock as the frequency source for both the VLBI equipment and the IGS stations. Table 2 summarizes the situation during the Cont08 experiment. Only the IGS stations ONSA and CONT were capable of time synchronization, where CONT data was unfortunately lost during Cont08 and thus an evaluation of time-transfer was not possible. In order to assess the correctness of the VLBI derived clock difference frequencies we have used comparisons with GPSCP solutions for the seven common clock cases. Due to the global distribution of the stations Precise Point Positioning (PPP) derived station clocks, by means of GIPSY/OASIS II [17] and NRCANPPP [18], were differentiated in order to create matching clock differences to the Onsala H-maser. NRCANPPP is capable of continuously processing arbitrary long time series without introducing day boundary offsets. GIPSY processing available to this study handles only $24 \mathrm{~h}$ batches and was used to study daily variations. The last column in Table 2 describes the results for the NRCAN case. The Onsala-Wettzell baseline, using the WTZZ receiver and the VLBI network solution, showed best agreement and stability. The link difference has a significant frequency offset of about 5e-16. This offset can be attributed to either technique, the absence of integer ambiguity resolution in NRCANPPP and frequency biases introduced by the VLBI day boundary fix. Day by day comparison with GIPSY results reveals significant frequency offsets between the solutions of maximal $\pm 2 \mathrm{e}-15$ and about 50ps RMS around the estimated lines.

\section{CONCLUSIONS}

Considering the equipment at Onsala as an example for a typical setup it is evident that VLBI time-transfer in its literal meaning is not possible at the time being without changes to the equipment and processing procedures. The correlator introduced biases and day-batch processing cause day boundary offsets that make characterization of long time series difficult. Link stabilities are easily over-estimated without careful balanced filter parameters. Further, potential Cont08 link stabilities of about 1e-15@1d could not match results reported in [11]. A practical use of VLBI for time-transfer will require a consistent calibration and a continuous correlation and analysis process. VLBI2010 with is wideband $24 / 7$ observations will improve the link noise and may make absolute time-transfer possible. 
Table 2. Cont08 IGS and clock equipment summary, network solution comparison with GPSCP NRCANPPP solutions. Onsala is the clock reference for both techniques. Trend estimates are in most cases significant. Green fields indicate best respective cases for Cont08.

\begin{tabular}{|c|c|c|c|c|c|c|c|c|c|c|}
\hline \multicolumn{7}{|c|}{ CONT08 Stations } & \multirow{2}{*}{\begin{tabular}{|c|} 
VLBI \\
AㄴㄴNN (@) 1, d]
\end{tabular}} & \multirow{2}{*}{ 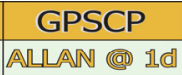 } & \multicolumn{2}{|c|}{ GPSCP-VLBI } \\
\hline VLBII & \multicolumn{2}{|c|}{ GNSS/TGS } & \multicolumn{3}{|c|}{ COnn!ñon:Clock } & GINSS IIIInneSyync & & & $\triangle T \mathbb{R} E \mathbb{N} \mathbb{D}$ & RMS \\
\hline HARTRAO & HRAO & (ASHTECH UZ-12) & YES & \multicolumn{2}{|l|}{ EFOS C } & NO & $1.9 e-15$ & 8.5e-16 & $3.8 \mathrm{e}-17$ & 260ps \\
\hline KOKEE & KOKB & (ASHTECH UZ-12) & YES & \multicolumn{2}{|l|}{ SigmaTau } & NO & $3.0 \mathrm{e}-15$ & $3.2 \mathrm{e}-15$ & $7.4 \mathrm{e}-17$ & 210ps \\
\hline MEDICINA & MEDI & (TRIMBLE 4000SSI) & YES & \multicolumn{2}{|l|}{ EFOS 4} & NO & $1.2 \mathrm{e}-14$ & $1.2 \mathrm{e}-14$ & $1.2 \mathrm{e}-15$ & $340 p s$ \\
\hline \multirow{2}{*}{ NYALES20 } & NYAL & (AOA BENCIMARK ACT) & \multirow{2}{*}{ YES } & \multirow{2}{*}{\multicolumn{2}{|c|}{ APL No2 }} & NO & \multirow{2}{*}{$6.8 e-15$} & $6.5 e-15$ & $-4.5 e-16$ & 110ps \\
\hline & NYA1 & (AOA BENCHMARK ACT) & & & & NO & & $6.8 e-15$ & $-6.6 e-16$ & 140ps \\
\hline ONSALA60 & ONSA & (JAVAD GGD) & YES & \multicolumn{2}{|l|}{ CH1-75A } & $\begin{array}{l}\text { YES } 40 / 7218 \text { is TAI } \\
\text { traceable via UTC(SP) }\end{array}$ & \multicolumn{4}{|c|}{ SOLVE/GPSCP REFERENCE } \\
\hline SVETLOE & SVTL & (LEICA SR520) & NO & IEM CH1-80 & internal & NO & $2.8 e-15$ & $1.1 \mathrm{e}-12$ & no commc & clock \\
\hline \multirow{2}{*}{ TIGOCONC } & CONT & (Spententrin POI ARX) ) & YES & \multirow{2}{*}{ EFOS 24} & EFOS 24 & $\begin{array}{l}\text { YES } 40 / 8620 \text { is TAI } \\
\text { traceable via UTCCTC }\end{array}$ & \multirow{2}{*}{$4.3 e-15$} & \multicolumn{3}{|c|}{ no GPS data available } \\
\hline & CONZ & (TPS E_GGD) & NO & & internal & NO & & $6.1 \mathrm{e}-9$ & no comms & clock \\
\hline \multirow{2}{*}{ TSUKUB32 } & TSK2 & (TRIMBLE 5700) & NO & \multirow{2}{*}{$\begin{array}{l}\text { Anritsu } \\
\text { RH401A }\end{array}$} & HP 5071A & \multirow{2}{*}{ NO Site maintains } & \multirow{2}{*}{$1.2 \mathrm{e}-13$} & $8.2 e-14$ & \multicolumn{2}{|c|}{ no common clock } \\
\hline & TSKB & (AUA BENCHMARK ACT) & NO & & HP 5071A & & & $6.5 e-14$ & \multicolumn{2}{|c|}{ no common clock } \\
\hline WESTFORD & WES2 & (ASHTECH UZ-12) & NO & $\begin{array}{l}\text { APL No3 or } \\
\text { No4 }\end{array}$ & internal & NO & $7.1 \mathrm{e}-15$ & $8.4 \mathrm{e}-14$ & \multicolumn{2}{|c|}{ no common clock } \\
\hline \multirow{3}{*}{ WETTZELL } & WTZR & (LEICA GRX1200GGPRO) & \multirow{3}{*}{ YES } & \multirow{3}{*}{\multicolumn{2}{|c|}{ EFOS 18}} & \multirow{3}{*}{$\mid \begin{array}{l}\text { NO } 40 / 4418 \text { is IAI } \\
\text { tracebale via UTC(IFAG) }\end{array}$} & \multirow{3}{*}{$1.2 e-15$} & $1.9 e-14$ & $8.9 e-15$ & 1970ps \\
\hline & WTZS & (Septentrio POLARX2) & & & & & & $8.2 e-11$ & \multicolumn{2}{|c|}{ phase jump in GPS } \\
\hline & WTZZ & (TPS E_GGD) & & & & & & $6.2 \mathrm{e}-16$ & $4.8 e-16$ & $70 p s$ \\
\hline ZELENCHK & ZECK & (ASHTECH Z-XII3) & NO & $\mathrm{CH} 1-80$ & internal & NO & $4.0 e-14$ & $4.1 \mathrm{e}-10$ & \multicolumn{2}{|c|}{ no common clock } \\
\hline
\end{tabular}

\section{ACKNOWLEDGMENTS}

This work has been performed as part of the Swedish National Metrology Program, program owner VINNOVA Swedish Agency for Innovation Systems. We would like to acknowledge the work of the following organization/individuals: Karl-Åke Johansson at Onsala Space Observatory (CTH), Per Jarlemark at SP, National Resources Canada for the NRCan GPS PPP software, and the Open Source Community at large.

\section{REFERENCES}

[1] http://deepspace.jpl.nasa.gov/dsn/

[2] Hurd et al., "Submicrosecond Comparison of Intercontinental Clock Synchronization by VLBI and the NTS Satellite", DSN Progress Report 42-49, November and December 1978

[3] Clark et al., "Precise Clock Synchronization via Very Long Baseline", Proc. $9^{\text {th }}$ PTTI, page 127, 1977

[4] Spencer et al., "Comparison of VLBI, TV, and Travelling Clock Techniques for Time Transfer", Proc. $13^{\text {th }}$ PTTI, page 231,1981

[5] Johnston et al., “ Precise Time Transfer Using MKIII VLBI Technology”, Proc. 15 $5^{\text {th }}$ PTTI, page 443, 1983

[6] Ward S.C., "Using GPS and VLBI Technology to Maintain 14 Digit Syntonization", Proc. $16^{\text {th }}$ PTTI, p. 447, 1984

[7] Hama S. et al. , "Japan-US time comparison experiment for realizing better than 1-ns accuracy by using a radio interferometric technique", IEEE Transactions on Instrumentation and Measurement, page 640, 1989

[8] Yoshino et al. , "Development of VLBI time transfer system toward $0.1 \mathrm{nsec}$ accuracy using transportable ground terminal for calibration", Proc. IEEE IMTC/94, page 806 vol.2, 1994

[9] Yoshino et al. , "The first comparative experiment of VLBI and two-way time transfer with better than 1 nsec precision", Proc. IEEE IMTC/94, page 808 vol.2,1994

[10] Takiguchi et al., "VLBI Measurements for Time Transfer between Time and Frequency Laboratories", AGU, Fall Meeting 2007, poster \#G33A-0903

[11] Takiguchi et al. , "VLBI Measurements for Time and Frequency Transfer", Proc EFTF2008, 2008

[12] http://www.haystack.mit.edu/geo/vlbi td/2010

[13] Niell a. et al.,"VLBI2010: A Vision for Future Geodetic VLBI", IAG, ZARAGOZA 2005

[14] Ma, C., Sauber, J. M., Bell, J. L., Clark, T. A., Gordon, D., Himwich, W. E.: "Measurement of horizontal motions in Alaska using Very Long Baseline Interferometry”. Journal of Geophysical Research, 95(B13), 21991-22011, 1990.

[15] W. Schlüter, D. Behrend, "The International VLBI Service for Geodesy and Astrometry (IVS): current capabilities and future prospects", Journal of Geodesy, Vol. 81, Nos. 6-8, pp. 379-387, June 2007.

[16] http://ivscc.gsfc.nasa.gov/program/cont08/

[17] Webb, F. H., and Zumberge, J. F, “An introduction to GIPSY/OASIS II.” JPLPublication D-11088, 1993.

[18] D. Orgiazzi, P. Tavella, F. Lahaye, "Experimental Assessment of the Time Transfer Capability of Precise Point Positioning (PPP)“, Proc. of the Joint IEEE Intl. FCS and the PTTI, Vancouver, Canada. 2005 